\title{
Effect of Probiotics and Prebiotics on Oral Health
}

\author{
Goutam Nanavati ${ }^{1} \quad$ T. Prasanth ${ }^{1} \quad$ Manab Kosala ${ }^{1}$ Sujit K. Bhandari ${ }^{2}$ Pamil Banotra ${ }^{1}$
}

\author{
${ }^{1}$ Department of Periodontology, Armed Forces Medical College, \\ Pune, Maharashtra, India \\ 2Department of Oral and Maxillofacial Surgery, Armed Forces \\ Medical College, Pune, Maharashtra, India
}

\begin{abstract}
Address for correspondence T. Prasanth, MDS, Department of Periodontology, Armed Forces Medical College, Wanowrie, Pune 411040, Maharashtra, India (e-mail: tprasanthavin@gmail.com).
\end{abstract}

\begin{abstract}
Keywords

- probiotics

- prebiotics

- periodontitis

- halitosis

Probiotics are the living microorganisms that have been commonly used in the prevention of gastrointestinal disorders. In recent times, probiotic and prebiotics have been used to assess and develop a natural balance of the microbial flora in the respiratory tract and the oral cavity as an adjunct therapy. They are known to augment the existing microbial flora that is beneficial to the host. Prebiotics are nondigestible food ingredients that help in increasing populations of probiotic bacteria. Recent studies have shown that probiotics help in active reduction in gingivitis, dental caries, periodontitis, and halitosis by replacing the harmful oral species, by means of utilizing abundant health-associated oral microbial species. Additionally, the nitrate-reducing bacteria have shown promising effect in improving efficiency of probiotic strains to accentuate oral health benefits. Probiotics along with prebiotics effectively alter the host-microbial interface by achieving homeostasis in multifactorial diseases such as periodontal diseases and oral malodor. The aim of the review is to collate the existing information available on use of probiotics and prebiotics in oral cavity.
\end{abstract}

\section{Introduction}

Probiotics are live nonpathogenic microorganisms administered to improve microbial balance the host. ${ }^{1}$ Probiotics together with prebiotics confer synergistic effects. The term "prebiotics" was first introduced by Gibson and Roberfroid in 1995, where they used Bifidobacterium and Lactobacillus to deliver health-promoting benefits by modulation of human colon. ${ }^{2}$ Use of microbiome therapy in homeostasis of oral cavity is a relatively new concept.

Since the conceptualization of probiotics, they have been described in many different ways. In recent times, use of probiotics is not restricted only to the gut and intestine, but also in oral cavity, female urogenital tract, and respiratory tract. ${ }^{3}$ Thus, probiotics have been defined as "live microorganisms that, when administered in adequate amounts, confer a health benefit on the host."

published online February 8, 2021
DOI https://doi.org/ $10.1055 / \mathrm{s}-0040-1722523$ ISSN 2321-1482.
Prebiotics are the compounds in food that add to the growth and/or increase the activity of the probiotic organisms in the host. These prebiotics are basically fibrous compounds that are nondigestible. Thus, they pass through upper gastrointestinal (GI) tract undigested, acting as the substrate for the advantageous microorganisms, helping in their growth and enhanced biological activity. ${ }^{3}$ One need not take prebiotic for probiotic organisms to act, but together prebiotics make probiotic organisms more effective.

Periodontitis is a chronic inflammatory disease characterized by connective tissue destruction and alveolar bone resorption. ${ }^{5}$ Most of the therapies are directed toward arresting the disease progression, regaining the lost periodontal tissue, and balancing the host response to the virulence factor of the pathogenic organisms. Socransky in 1998 explained different bacterial complexes, related to periodontium in

(C)2021. Bhojia Dental College and Hospital affiliated to Himachal Pradesh University.

This is an open access article published by Thieme under the terms of the Creative Commons Attribution-NonDerivative-NonCommercial-License, permitting copying and reproduction so long as the original work is given appropriate credit. Contents may not be used for commercial purposes, or adapted, remixed, transformed or built upon. (https://creativecommons.org/licenses/by-nc-nd/4.0/).

Thieme Medical and Scientific Publishers Pvt. Ltd. A-12, 2nd Floor, Sector 2, Noida-201301 UP, India 
health and disease. Red and orange complex bacteria are the most common microorganisms associated with periodontal disease. $^{6}$

Halitosis is the presence of foul and unpleasant odor originating from the oral cavity, primarily caused by Fusobacterium nucleatum, Porphyromonas gingivalis, P. intermedia, and Treponema denticola. It is due to the production of volatile sulfur compounds (VSCs). ${ }^{7}$

Dental caries is an infectious microbial disease resulting from production of organic acids due to the fermentation of carbohydrate. These acids cause demineralization of hard tissue of tooth, resulting in destruction and loss of tooth structure. $^{8}$

All the above conditions have microbial plaque as a common etiological factor. Probiotics play an important role in modifying the local environment of oral cavity and reducing the dysbiosis. Thus, they help in maintaining homeostasis. ${ }^{9}$ Keeping in mind the growing resistance to the antibiotics and frequent recolonization of the organisms in dental plaque, use of probiotics in oral cavity is on the rise. ${ }^{10}$

\section{History of Probiotics}

Probiotics were introduced as early as 1908 by Elie Metchnikoff where he demonstrated the health benefits and increased lifespan in Bulgarian population attributing to consumption of fermented dairy products. ${ }^{1}$ Being the pioneer of given field, he is referred to as "grand father of modern probiotics." Dairy products such as milk, curd, and buttermilk are a regular part of diet in India. Medieval literature on dietetics "Kshemakutuhalam" emphasizes the importance of including components of milk products in diet, calling buttermilk as "elixir of gods." ${ }^{11}$ Use of sour milk as a cure for upset stomach has been documented in Bible also.

Around early 1900s, Henry Tissier, a French pediatrician, also conducted a study on probiotic organisms. He observed that children suffering from diarrhea when ingested with $\mathrm{Y}$ shaped "bifid" bacteria presented with healthy gut flora. ${ }^{12}$ "Yakult," a commercially available probiotic drink consisting of "Lactobacillus" species, was launched in Japan in $1930 .{ }^{13}$ The term "probiotics" became popular after Lilly and Stillwel used it for the first time in 1965 to address health effect of many protozoal species. ${ }^{14}$ Lactobacillus acidophilus (Hull et al) and Bifidobacterium bifidum (Holcombh et al) were the first probiotic microorganisms used for research purpose. ${ }^{15,16}$

Haukioja et al are considered as early pioneers in studying the interaction of probiotics in oral cavity. They analyzed the adhesion of lactobacilli and bifidobacterial species to hydroxyapatite crystals that were coated with saliva (in vitro). ${ }^{17}$

\section{Probiotics}

Probiotics is a term derived from Greek language, which means "for life." They include many microbial species and strains. ${ }^{18}$ Commonly studied probiotics applied in the food and health areas are given in - Table 1.

Several studies (both clinical and experimental) have proven that certain GI tract bacteria, that is, Lactobacillus and Bifidobacterium species, can control and manipulate the growth of microorganisms causing oral diseases. For probiotics to confer its benefits to host, the food should contain at least $10^{6}-10^{7}$ live organisms per $\mathrm{g}$ or $\mathrm{mL}$ of product at the time of consumption, general frequency of consumption of probiotics being five billion to 10 billion colony-forming units per day. ${ }^{19}$

\section{Guidelines for Use of Probiotics}

FAO/WHO in 2002 issued guidelines for the evaluation of probiotics for use in food products.

1. Probiotic organisms must be a live organism.

2. The organism must be identified up to species level.

3. It should have a proven safety data.

4. When used in a defined value and delivery system, must have a physiological effect on the host.

5. When used as food additive, must be biologically and genetically stable.

6. It should be economical.

7. It should maintain long-term stability on storage (adequate shelf life).

8. It should be nonpathogenic and nonhazardous.

9. It should be able to influence local metabolic activity. ${ }^{20}$

Table 1 Commonly used probiotics

\begin{tabular}{|c|c|c|}
\hline Species & Strains & References \\
\hline Lactobacillus & $\begin{array}{l}\text { L. acidophilusL. amylovorus, L. casei, L. cebollobiosus, L. crispatus, } \\
\text { L. curvatus, L. delbrueckii subsp. bulgaricus, L. fermentum, L. gasser, L. helveticus, } \\
\text { L. johnsonii, L. lactis, L. paracasei, L. pentosus, L. plantarum, L. reuteri, L. rhamnosus, } \\
\text { L. salivarius }\end{array}$ & $25,52-55$ \\
\hline Bifidobacterium & $\begin{array}{l}\text { B. adolescentis, B. animalis, B. bifidum, B. breve, B. infantis, B. lactis, B. longum, } \\
\text { B. thermophilus }\end{array}$ & $25,53,54$ \\
\hline Other species of lactic acid bacteria & $\begin{array}{l}\text { Enterococcus faecium, Lactococcus lactis, L. lactis subsp. cremoris, Leuconstoc } \\
\text { mesenteroides, Pediococcus acidilactici, Streptococcus salivarius subsp. thermophilus, } \\
\text { Streptococcus thermophilus, } \\
\text { S. intermedius, S. diaacetylactis, Sporolactobacillus inulinus }\end{array}$ & 53,54 \\
\hline Nonlactic acid bacteria & $\begin{array}{l}\text { Bacillus cereus var. toyoi, Bacillus coagulans, Bacillus clausii, Saccharomyces cerevisiae, } \\
\text { Saccharomyces boulardii, Escherichia coli strain nissle, Propionibacterium freudenreichii }\end{array}$ & $54-56$ \\
\hline
\end{tabular}




\section{Mechanism of Action of Probiotics}

In recent times, probiotics have emerged as substitute to antibiotics to help reduce various oral conditions such as periodontitis, dental caries, and halitosis. The proposed mechanism of action can be broadly classified under direct and indirect. Direct mode of action comprises of probiotic organisms having effect on the pathogenic organisms itself (-Fig. 1). The indirect mode of action is the one with probiotic organisms modulating the host response toward the pathogens.

\section{Direct Action of Probiotics}

L. reuteri has bactericidal action by producing ethanol, reuterin, and reutericyclin. It also helps in production of cobalamin and folate in the gut of host (-Fig. 1).

L. jensenii, L. johnsonii, and B. bifidum damage the bacterial cell walls by releasing hydrogen peroxide. Many other probiotics produce bacteriocin enzyme, which destroys the pathogen. At the same time, the enzymatic biosurfactants secreted by the probiotic organisms help reduce adhesion of the bacteria to each other as well as to the plaque or/and tooth surface. ${ }^{21-24}$

\section{Indirect Action of Probiotics}

- Modulation of systemic immune response in favor of the host

- Affect local immune response

- Production of antioxidants or act as an antioxidant

- Reduction of pathogen associated proinflammatory cytokine production

- Prevention of plaque formation by neutralization of free electron

- Upregulation of epithelial barrier integrity and mucous production $^{21-23}$

\section{Prebiotics}

The term was coined by Gibson and Roberfoid in 1995, where they defined prebiotics as "the substrate that is selectively utilized by host microorganisms conferring a health benefit." 25 They are the food ingredients that are relatively not digested by host digestive system, which stimulate growth and activity of the probiotic organisms.
The most commonly used prebiotics are insulin type fructans, fructo-oligosaccharide, galacto-oligosaccharide, and lactose. ${ }^{26}$

Characteristic features of ideal prebiotic:

- They are neither hydrolyzed nor absorbed by mammalian enzymes or tissue.

- They selectively promote growth of certain probiotic species.

- They should be able to alter intestinal microbial flora and its activity.

- They should be able to modify systemic aspects of host defense system. ${ }^{27}$

\section{Symbiosis}

Symbiosis is a mutually beneficial relationship between different groups. In this scenario, the term is used for the commercially available product containing both probiotic and prebiotic component. Due to this combination, the survival rate of the probiotic organism increases, thus enhancing the chances of effective action of probiotic bacteria ${ }^{28}$ for example, Bifido bacterial species in conjugation with fructo-oligosaccharide and Lactobacillus species in conjugation with lactulose. This symbiosis helps improve the survivability and boost the growth of these probiotics. ${ }^{27}$

\section{Probiotics in Oral Health}

The use of probiotics in enhancing oral health dates back to early 90 s.

\section{Probiotics and Periodontal Disease}

Probiotic organisms act as a supportive tool with binary benefits to manage periodontal inflammation. Primarily by causing dysbiosis, they result in competitive inhibition of periodontal pathogen thereby reducing the microbial load. ${ }^{29}$

Secondarily by invigorating dendritic cells, they cause increase in the number helper $\mathrm{T}$ cells that modulate host immune response toward microorganisms present with the added benefit of least periodontal destruction during the process..$^{30}$ Bacteriocins secreted by $L$ salivarius, $L$ gasseri, and catalase secreted by Weissella cibaria cause an alteration in aggregation of bacteria responsible for plaque formation. ${ }^{30}$ Streptococcal strains when used as probiotics, as

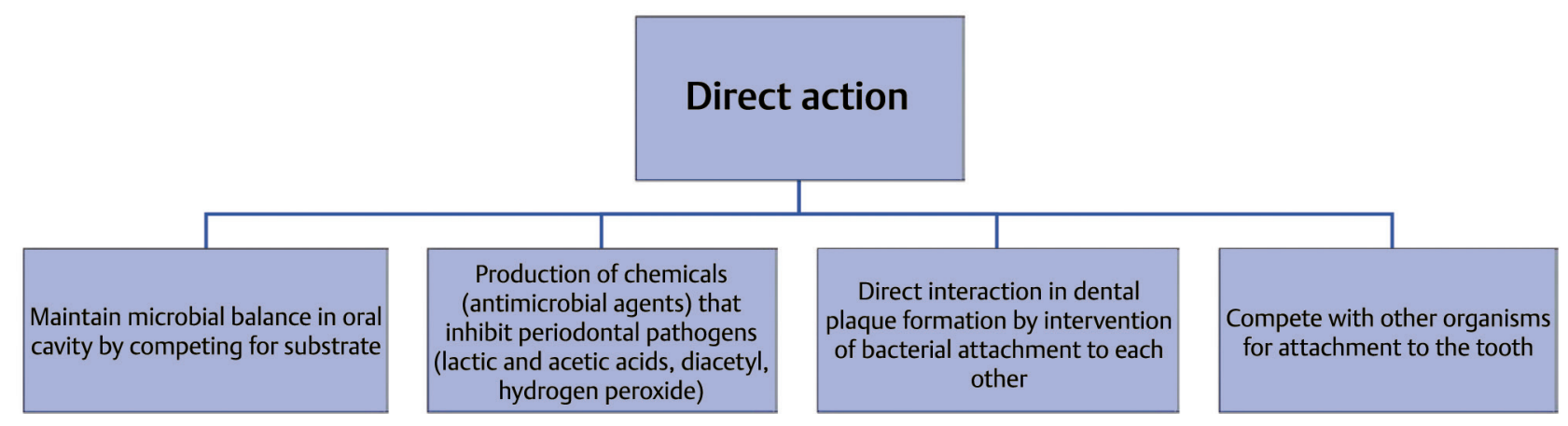

Fig. 1 Direct action of probiotics in oral cavity. 
an adjunct to scaling, and root planning in chronic periodontitis patient show a significant reduction in plaque score along with reduction in red complex bacteria. ${ }^{31}$ Reutrin and reutericyclin enzymes secreted by $L$. reuteri inhibit the growth of pathogens by preventing the secretion the proinflammatory mediators. As a result, they exert an anti-inflammatory effect. ${ }^{32} L$. gasseri and $L$. fermentum are more prevalent in healthy periodontium as compared with patients with chronic periodontitis, due to the production of hydrogen peroxide and bacteriocin. ${ }^{33}$ Bifidobacterial species have shown competitive inhibition of pigment forming bacteria in oral cavity because of its utilization of vitamin K as substrate. ${ }^{34}$

It was observed, when L. plantarum (HKL-137) was used during supportive therapy for chronic periodontitis for 12 weeks; there was reduction in probing depth as compared with the placebo group..$^{35}$ L. rhamnosus and B. lactis (BB-12) affect the numbers of $P$. gingivalis and A. actinomycetemcomitans by significantly reducing their load in oral cavity and help in promoting gingival health. ${ }^{36}$

\section{Probiotics and Halitosis}

Halitosis is the general term used to describe any disagreeable odor of expired air, regardless of its origin. ${ }^{37}$ It is an unpleasant odor most commonly caused by Fusobacterium nucleatum, $P$. gingivalis, $P$. intermedia, and $T$. denticola. The microbiota causes putrefactive metabolism of amino acids producing VSCs (hydrogen sulfide, methyl mercaptan, dimethyl sulfide). ${ }^{38}$

Tongue acts as a niche for accumulation of microbiota due to the presence of multiple papillae on its dorsal surface. Probiotics help to reduce the dysbiosis of microbiota on the tongue especially the posterior part, which is difficult to clean..$^{39}$

L. salivarius WB21 strain when administered orally as tablet, thrice a day, showed a significant decrease in organoleptic score due to decline in the concentration of VSCs..$^{40}$

$W$. cibaria when used as a probiotic agent demonstrated a reduction in $\mathrm{H}_{2} \mathrm{O}_{2}$ production, due to inhibition of $F$. nucleatum. ${ }^{41}$ Streptococcus salivarius (K12 strain), one of the pioneer probiotic strain, secrets bacteriocin, which when consumed as lozenges helps reduce Solobacterium moorei, thereby reducing oral mal odor. ${ }^{42}$

L. reuterin has also been beneficial in promoting oral health and reducing oral mal odor. By downregulation of enzyme methionine $\gamma$ lyase is produced by $F$. nucleatum and P. gingivalis. ${ }^{43}$

\section{Probiotics and Dental Caries}

Caries is an infectious microbial disease of oral cavity caused by fermentation of carbohydrates by bacteria resulting in dissolution of the mineral content of the tooth structure. The main organism responsible is $S$. mutans. There is increased prevalence of dental caries, especially in children due to increase in consumption of refined sugar and inadequate cleaning habits. ${ }^{44}$

L. rhamnosus GG strain, L. reuteri, and Bifidobacterium lactis BB-12 strain when used as probiotics have shown significant reduction in the number of S. mutans. They potentially deter the microbiota of dental plaque and reduce the adhesion of bacteria to the tooth surface, resulting in reduced incidence of dental caries. When multispecies or multistrains of probiotic organisms are used, there is a considerable decline in the cariogenic bacteria. ${ }^{45}$ Similarly, L. rhamnosus $1 \mathrm{~b} 21$ strain, when used in milk along with fluoride, produces the same result. ${ }^{46}$

\section{Commercially Available Probiotics}

Probiotics used to elevate oral health and treat dental conditions are commonly available as lozenges, toothpaste, chewing gums, or mouthwash.

\section{ProBiora Health}

It is designed to improve in general oral health of humans as well as pets. It is a multistrain blend of Streptococcus strains, S. oralis KJ3, S. uberis KJ2, and S. rattus JH145. ${ }^{47}$

\section{Gum PerioBalance}

It is one of the pioneer formulations for treating periodontal disease. It has a combination of two strains of $L$. reuteri to act against periodontal pathogens. ${ }^{48}$

\section{Blis Technologies}

It includes the formulation of two strains of BLIS K12 and BLIS M18 probiotics that are native to oral cavity. They control halitosis along with having beneficial effect on throat and nasal health. ${ }^{49}$

\section{PeriBiotic}

It is a toothpaste containing $L$. paracasei probiotic that reduces periodontal pathogens. ${ }^{48}$

\section{NatureWise}

This probiotic preparation is commercially available in the form of chewable tablets. It contains 12 different types of probiotic strains: $L$ casei subsp. casei, L. paracasei Lpc37, L. plantarum, L. reuteri, L. salivarius, S. salivarius K12, S. salivarius M18, B. lactis $\mathrm{Bl}-04$, L. rhamnosus GG, B. breve $\mathrm{Bb}-03, B$. infantis, and $S$. thermophilus. The aim is to have a protective effect on the oral cavity by altering the pathogenic bacterial colonies.

\section{Prodentis}

This probiotic lozenge is a blend of two Lactobacillus reuteri strains containing a minimum of $1 \times 108 \mathrm{CFU}$ for each of the strains DSM 17938 and ATCC PTA 5289. ${ }^{48}$

\section{Safety Concerns}

FDA classifies probiotic organisms as generally regarded as safe. But due to the increase in probiotic supplementation of various food products in the recent years, their safety standards still need to be investigated and verified. There is still a reasonable likelihood of bacteremia in critically ill or immunocompromised patients as probiotics are live organisms. ${ }^{50} \mathrm{It}$ 
may also present as a potential risk for people with lactose intolerance or hypersensitivity to milk products.

\section{Future of Probiotics}

With an increasing threat of microorganisms developing resistance to the known antibiotics, probiotics have emerged as an improved and innovative solution to the above-stated problem. Probiotics are way ahead of their conventional counter products with an added advantage of minimal side effects. There is a change in lifestyle and feeding habits of people across the globe, indicating an upsurge of oral diseases proportional to other lifestyle diseases attributing to the same factor. These natural strains of probiotic organisms isolated from dairy products, for example, curd, buttermilk can be used on regular basis for management and maintenance of dental diseases and oral health, respectively.

"Designer probiotics," a relatively new concept of genetically modified microorganisms which target a specific pathogen, can be utilized as a novel tool to act against the severe oral diseases. These strains achieve the desired effect by disruption of biofilm and formation of enzymes such as bacteriocins. They can also be designed to endure high amount of stress, thus improving their sustainability in oral cavity. Successful use of probiotics in dentistry can pave a way to advanced treatment options and therapy modules to augment the oral health in general. ${ }^{51}$

\section{Conclusion}

The aim of this review was to find out the existing literature regarding the use of probiotics in oral cavity. Probiotics as we know are useful in maintaining the microbial balance in the human body. Use of probiotics in oral cavity is an emerging concept. They play a significant role in clinical management of halitosis, dental caries, and periodontal diseases. These studies suggest that probiotics can reduce the load of pathogenic organisms as well as restore the host microbiota. To explore the potential of prebiotics, further studies are required to understand the ability of the probiotic organism to survive, grow, and have a therapeutic effect. Also we need well designed, long-term follow-up studies to fix the dosages and schedules of administration, interaction with various substrate, interpretation of oral health risks in case of long-term use of the probiotic organisms; so as to emphasize and practice the same accordingly.

\section{Conflict of Interest}

None declared.

\section{References}

1 Metchnikoff E, Optimistic Studies. New York: Putman's Sons; $1908 ; 161-183$

2 Gibson GR, Roberfroid MB. Dietary modulation of the human colonic microbiota: introducing the concept of prebiotics. J Nutr 1995;125(6):1401-1412

3 Gibson GR, Hutkins R, Sanders ME, et al. Expert consensus document: The International Scientific Association for
Probiotics and Prebiotics (ISAPP) consensus statement on the definition and scope of prebiotics. Nat Rev Gastroenterol Hepatol 2017;14(8):491-502

4 Food and Agriculture Organization and World Health Organization Expert Consultation, Evaluation of health and nutritional properties of powder milk and live lactic acid bacteria. Córdoba, Argentina: Food and Agriculture Organization of the United Nations and World Health Organization; 2001

5 Page RC, Kornman KS. The pathogenesis of human periodontitis: an introduction. Periodontol 2000 1997;14(1):9-11

6 Socransky SS, Haffajee AD, Cugini MA. Smith C, Kent RL Jr. Microbial complexes in subgingival plaque. J Clin Periodontol 1998;25(2):134-144

7 Persson S, Edlund MB, Claesson R, Carlsson J. The formation of hydrogen sulfide and methyl mercaptan by oral bacteria. Oral Microbiol Immunol 1990;5(4):195-201

8 Featherstone JD. The continuum of dental caries-evidence for a dynamic disease process. J Dent Res 2004;83 Spec No C(1_ suppl) :C39-C42

9 Allaker RP, Stephen AS. Use of probiotics and oral health. Curr Oral Health Rep 2017;4(4):309-318

10 Zambori C, Morvay AA, Sala C, et al. Antimicrobial effect of probiotics on bacterial species from dental plaque. J Infect Dev Ctries 2016;10(3):214-221

11 Kukkupuni SK, Shashikumar A, Venkatasubramanian P. Fermented milk products: probiotics of ayurveda. Journal of Medical Nutrition and Nutraceuticals. 2015;4(1):14-21

12 Tissier $\mathrm{H}$. The treatment of intestinal infections by the method of transformation of bacterial intestinal flora. CR Soc. Biol. 1906;60:359-361

13 Anukam KC, Reid G. Probiotics: 100 years (1907-2007) after Elie Metchnikoff's observation. Communicating current research and educational topics and trends in applied microbiology; 2007;1:466-474

14 Lilly DM, Stillwell RH. Probiotics: growth-promoting factors produced by microorganisms. Science 1965;147(3659):747-748

15 Hull RR, Roberts AV, Mayes JJ. Survival of Lactobacillus acidophilus in yoghurt. Australian Journal of Dairy Technology 1984;39(4):164

16 Holcomb JE, Frank JF, Mc Gregor JU. Viability of Lactobacillus acidophilus and Bifidobacterium bifidum in softserve frozen yogurt. 1991;26:4-5

17 Haukioja A, Yli-Knuuttila H, Loimaranta V, et al. Oral adhesion and survival of probiotic and other lactobacilli and bifidobacteria in vitro. Oral Microbiol Immunol 2006;21(5):326-332

18 Isolauri E. Probiotics in human disease. Am J Clin Nutr 2001;73(6):1142S-1146S

19 Shah NP. Functional cultures and health benefits. Int Dairy J 2007;17(11):1262-1277

20 Joint FAO/WHO Expert Committee on Food Additives, Joint FAO/WHO Expert Committee on Food Additives. Meeting, World Health Organization. Evaluation of Certain Food Additives and Contaminants: Fifty-seventh Report of the Joint FAO/WHO Expert Committee on Food Additives. World Health Organization; 2002 Dec 13

21 Mahasneh SA, Mahasneh AM. Probiotics: a promising role in dental health. Dent J (Basel) 2017;5(4):26-36

22 Agarwal G, Ingle NA, Kaur N, Yadav P, Ingle E, Charania Z. Probiotics and oral health: a review. J Int Oral Health 2015;7(10):133-136

23 Meurman JH. Probiotics: do they have a role in oral medicine and dentistry? Eur J Oral Sci 2005;113(3):188-196

24 Haukioja A. Probiotics and oral health. Eur J Dent 2010;4(3):348-355

25 Bustamante M, Oomah BD, Rubilar M, Shene C. Effective Lactobacillus plantarum and Bifidobacterium infantis encapsulation with chia seed (Salvia hispanica L.) and flaxseed 
(Linum usitatissimum L.) mucilage and soluble protein by spray drying. Food Chem 2017;216:97-105

26 Pandey KR, Naik SR, Vakil BV. Probiotics, prebiotics and synbiotics- a review. J Food Sci Technol 2015;52(12):7577-7587

27 Maftei NM. Probiotic, Prebiotic and Synbiotic Products in Human Health. In: Frontiers and New Trends in the Science of Fermented Food and Beverages. London, IntechOpen;2019

28 Sekhon BS, Jairath S. Prebiotics, probiotics and synbiotics: an overview. Journal of Pharmaceutical Education and Research. 2010;1(2):13-36

29 Saha S, Tomaro-Duchesneau C, Tabrizian M, Prakash S. Probiotics as oral health biotherapeutics. Expert Opin Biol Ther 2012;12(9):1207-1220

30 Bose BB. Probiotics-promotes periodontal health?-An insight. International Journal of Dental Sciences and Research. 2013;1(3):67-70

31 Laleman I, Yilmaz E, Ozcelik O, et al. The effect of a streptococci containing probiotic in periodontal therapy: a randomized controlled trial. J Clin Periodontol 2015;42(11):1032-1041

32 Szkaradkiewicz AK, Stopa J, Karpiński TM. Effect of oral administration involving a probiotic strain of Lactobacillus reuteri on pro-inflammatory cytokine response in patients with chronic periodontitis. Arch Immunol Ther Exp (Warsz) 2014;62(6):495-500

33 Gupta G. Probiotics and periodontal health. J Med Life 2011; $4(4): 387-394$

34 Hojo K, Nagaoka S, Murata S, Taketomo N, Ohshima T, Maeda N. Reduction of vitamin $\mathrm{K}$ concentration by salivary Bifidobacterium strains and their possible nutritional competition with Porphyromonas gingivalis. J Appl Microbiol 2007;103(5):1969-1974

35 Iwasaki K, Maeda K, Hidaka K, Nemoto K, Hirose Y, Deguchi S. Daily intake of heat-killed Lactobacillus plantarum L-137 decreases the probing depth in patients undergoing supportive periodontal therapy. Oral Health Prev Dent 2016;14(3):207-214

36 Alanzi A, Honkala S, Honkala E, Varghese A, Tolvanen M, Söderling E. Effect of Lactobacillus rhamnosus and Bifidobacterium lactis on gingival health, dental plaque, and periodontopathogens in adolescents: a randomised placebo-controlled clinical trial. Benef Microbes 2018;9 (4):593-602

37 Aydin M, Harvey-Woodworth CN. Halitosis: a new definition and classification. Br Dent J 2014;217(1):E1-E10

38 Niles HP. Advances in Mouth Odor Research. Bad Breath: A Multidisciplinary Approach; 1996:55-69

39 Gurpinar B, Yildirim G, Kumral TL, et al. A simple method to reduce halitosis; tongue scraping with probiotics. J Breath Res 2019;14(1):016008

40 Suzuki N, Yoneda M, Takeshita T, Hirofuji T, Hanioka T. Induction and inhibition of oral malodor. Mol Oral Microbiol 2019;34(3):85-96
41 Kang MS, Kim BG, Chung J, Lee HC, Oh JS. Inhibitory effect of Weissella cibaria isolates on the production of volatile sulphur compounds. J Clin Periodontol 2006;33(3):226-232

42 Wescombe PA, Heng NC, Burton JP, Tagg JR. Something old and something new: an update on the amazing repertoire of bacteriocins produced by Streptococcus salivarius. Probiotics Antimicrob Proteins 2010;2(1):37-45

43 Fujiwara N, Murakami K, Nakao M, et al. Novel reuterin-related compounds suppress odour by periodontopathic bacteria. Oral Dis 2017;23(4):492-497

44 Tinanoff N, Baez RJ, Diaz Guillory C, et al. Early childhood caries epidemiology, aetiology, risk assessment, societal burden, management, education, and policy: Global perspective. International journal of Paediatric Dentistry 2019;29(3):238-248

45 Meurman JH, Stamatova I. Probiotics: contributions to oral health. Oral Dis 2007;13(5):443-451

46 Galdeano CM, de Moreno de LeBlanc A, Vinderola G, Bonet ME, Perdigón G. Proposed model: mechanisms of immunomodulation induced by probiotic bacteria. Clin Vaccine Immunol 2007;14(5):485-492

47 Duarte C, Al-Yagoob A, Al-Ani A. Efficacy of probiotics used as a periodontal treatment aid: a pilot study. Saudi Dent J 2019;31(1):143-147

48 Lawande S. Probiotics for management of periodontal disease: a novel therapeutic strategy. IOSR J Pharm 2012;2:41-46

49 Zupancic K, Kriksic V, Kovacevic I, Kovacevic D. Influence of oral probiotic Streptococcus salivarius K12 on ear and oral cavity health in humans: systematic review. Probiotics Antimicrob Proteins 2017;9(2):102-110

50 Cannon JP, Lee TA, Bolanos JT, Danziger LH. Pathogenic relevance of Lactobacillus: a retrospective review of over 200 cases. Eur J Clin Microbiol Infect Dis 2005;24(1):31-40

51 Chatterjee A, Bhattacharya H, Kandwal A. Probiotics in periodontal health and disease. J Indian Soc Periodontol 2011;15(1):23-28

52 Collins MD, Gibson GR. Probiotics, prebiotics, and synbiotics: approaches for modulating the microbial ecology of the gut. Am J Clin Nutr 1999;69(5):1052S-1057S

53 Holzapfel WH, Haberer P, Geisen R, Björkroth J, Schillinger U. Taxonomy and important features of probiotic microorganisms in food and nutrition. Am J Clin Nutr 2001; 73(2, Suppl):365S-373S

54 Markowiak P, Śliżewska K. Effects of probiotics, prebiotics, and synbiotics on human health. Nutrients 2017;9(9):1021-1051

55 Bustamante M, Oomah BD, Mosi-Roa Y, Rubilar M, Burgos-Díaz C. Probiotics as an adjunct therapy for the treatment of halitosis, dental caries and periodontitis. Probiotics Antimicrob Proteins 2020;12(2):325-334

56 Endres JR, Clewell A, Jade KA, Farber T, Hauswirth J, Schauss AG. Safety assessment of a proprietary preparation of a novel Probiotic, Bacillus coagulans, as a food ingredient. Food Chem Toxicol 2009;47(6):1231-1238 